\title{
Paclitaxel-loaded expansile nanoparticles enhance chemotherapeutic drug delivery in mesothelioma 3-dimensional multicellular spheroids
}

\author{
Hongyi Lei, MD, PhD, ${ }^{\text {a,b }}$ Sophie C. Hofferberth, MBBS, ${ }^{a}$ Rong Liu, MD, PhD, ${ }^{a}$ Aaron Colby, PhD, \\ Kristie M. Tevis, BS, ${ }^{\mathrm{c}}$ Paul Catalano, ScD, ${ }^{\mathrm{a}}$ Mark W. Grinstaff, $\mathrm{PhD},{ }^{\mathrm{c}}$ and Yolonda L. Colson, MD, $\mathrm{PhD}{ }^{\mathrm{a}}$
}

\begin{abstract}
Objectives: Intraperitoneal administration of paclitaxel-loaded expansile nanoparticles (Pax-eNPs) significantly improves survival in an in vivo model of malignant mesothelioma compared with conventional drug delivery with the clinically utilized Cremophor EL/ethanol (C/E) excipient. However, in vitro monolayer cell culture experiments do not replicate this superior efficacy, suggesting Pax-eNPs utilize a unique mechanism of drug delivery. Using a mesothelioma spheroid model, we characterized the mechanisms of enhanced tumor cytotoxicity leveraged by Pax-eNPs.
\end{abstract}

Methods: Human malignant mesothelioma (MSTO-211H) spheroids were coincubated for 24 hours with Oregon Green-conjugated paclitaxel dissolved in $\mathrm{C} / \mathrm{E}$ or loaded into eNPs. Oregon Green-paclitaxel uptake was measured as Oregon Green intensity via confocal microscopy and kinetics of tumor cytotoxicity were assessed via propidium iodide staining. Pharmacologic endocytotic inhibitors were used to elucidate mechanisms of eNP uptake into spheroids.

Results: Increased drug penetration and a 38-fold higher intraspheroidal drug concentration were observed 24 hours after MSTO-211H spheroids were treated with Oregon Green-conjugated paclitaxel loaded into eNPs compared with Oregon Green-conjugated paclitaxel dissolved in $\mathrm{C} / \mathrm{E}(P<.01)$. Macropinocytosis was the dominant endocytotic pathway of eNP uptake. Spheroids were more susceptible to paclitaxel when delivered via eNP, exhibiting more than twice the propidium iodine intensity compared with an equivalent paclitaxel- $\mathrm{C} / \mathrm{E}$ dose.

Conclusions: Compared with monolayer cell culture, the in vitro 3-D tumor spheroid model better reflects the superior in vivo efficacy of Pax-eNPs. Persistent tumor penetration and prolonged intratumoral release are unique mechanisms of Pax-eNP cytotoxicity. 3-D spheroid models are valuable tools for investigating cytotoxic mechanisms and nanoparticle-tumor interactions, particularly given the costs and limitations of in vivo animal studies. (J Thorac Cardiovasc Surg 2015;149:1417-25)

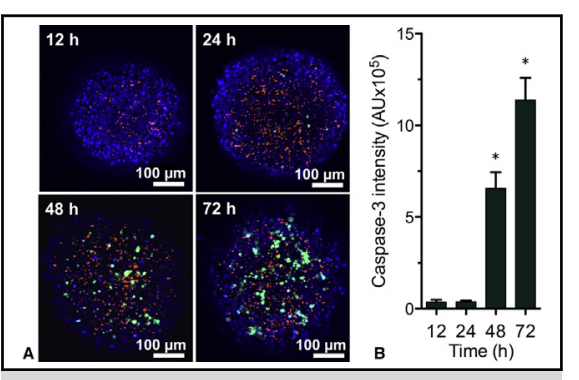

Paclitaxel-loaded expansile nanoparticles upregulate caspase-3 activity to enhance apoptosis within tumor spheroids.

\section{Central Message}

Monolayer cell culture models do not predict the superior in vivo efficacy of paclitaxel-loaded expansile nanoparticles (Pax-eNPs). Using an in vitro tumor spheroid model, we identified unique mechanisms of Pax-eNP enhanced cytotoxicity. Spheroid resistance to conventional drug exposure suggests this model can identify mechanisms of tumor cytotoxicity relevant to in vivo efficacy.

\section{Perspective}

We sought to address the significant differences observed between in vivo animal models and in vitro monolayer cell culture studies investigating the antitumor efficacy of local chemotherapeutic drug-delivery systems. An in vitro 3-dimensional tumor spheroid model was used to elicit unique mechanisms of enhanced tumor cytotoxicity leveraged by paclitaxel-loaded expansile nanoparticles (Pax-eNPs). Because the spheroid model more closely resembles the native tumor environment, these findings provide important clues to understanding the superior in vivo antitumor efficacy of Pax-eNPs. These results suggest that 3-dimensional spheroid models may be a clinically relevant platform to investigate mechanisms of tumor cytotoxicity and drug delivery.

See Editorial Commentary page 1426

\footnotetext{
From the Division of Thoracic Surgery, ${ }^{\text {a }}$ Department of Surgery, Brigham and Women's Hospital, Boston, Mass; Department of Anesthesiology, ${ }^{b}$ Zhujiang Hospital, Southern Medical University, Guangzhou, China; and Departments of Biomedical Engineering and Chemistry, ${ }^{\mathrm{c}}$ Boston University, Boston, Mass.

This work was supported by the Brigham and Women's Hospital International Mesothelioma Program, National Science Foundation (grant No. DMR-1006601) and Boston University's Nanomedicine Program and Cross-Disciplinary Training in Nanotechnology for Cancer (grant No. NIH R25 CA153955), and the Zhuangjiang Hospital Scholarship Program.

$\mathrm{HL}$ and $\mathrm{SCH}$ are co-first authors.
}

Read at the 94th Annual Meeting of The American Association for Thoracic Surgery, Toronto, Ontario, Canada, April 26-30, 2014.

Received for publication Dec 1, 2014; revisions received Feb 2, 2015; accepted for publication Feb 7, 2015; available ahead of print April 2, 2015.

Address for reprints: Yolonda L. Colson, MD, PhD, Division of Thoracic Surgery, Brigham and Women's Hospital, Harvard Medical School, 75 Francis St, Boston, MA 02115 (E-mail: ycolson@partners.org). $0022-5223 / \$ 36.00$

Copyright (C) 2015 by The American Association for Thoracic Surgery http://dx.doi.org/10.1016/j.jtcvs.2015.02.020 




Supplemental material is available online.

Nanoparticle (NP)-based drug-delivery systems have been used to successfully address many of the difficulties encountered during the administration of chemotherapeutic compounds. Encapsulation of drugs within NPs increases drug solubility; alters biodistribution; enhances pharmacokinetics through sustained release; protects sensitive drugs from low-pH environments or enzymatic alteration; and, in some cases, enables targeting of drugs to specific sites. ${ }^{1-5}$ Despite these significant advances, challenges remain in the evaluation of local chemotherapeutic drugdelivery systems, hindering their translation into the clinical setting. Importantly, the efficacy of chemotherapeutic delivery systems has been shown to be different for in vitro versus in vivo studies investigating the same agent. Previous studies performed by our group investigating the efficacy of paclitaxel-loaded expansile nanoparticles (Pax-eNPs) have shown that in vitro experiments with monolayer cell cultures do not predict the superior in vivo results demonstrated in animal models. For example, Colson and colleagues $^{6}$ demonstrated that Pax-eNP treatment of human intraperitoneal mesothelioma in an orthotopic in vivo xenograft model increases survival 2-fold compared with an equivalent dose of paclitaxel (Pax-C/E), even though PaxeNPs were less effective than Pax-C/E when investigated using monolayer-based in vitro cytotoxicity assays. ${ }^{6}$ These results suggest Pax-eNPs utilize additional mechanistic advantages in vivo that are not represented in traditional 2-dimensional (2-D) monolayer cultures. The inability to screen for efficacy of drug-delivery systems outside of whole organisms highlights the critical need to develop multicellular in vitro models to bridge the gap between conventional 2-D cell experiments and animal studies and further elucidate the mechanisms of tumor penetration and cytotoxicity of NP-based drug-delivery systems.

Multicellular spheroids are three-dimensional (3-D) in vitro microscale tissue analogs shown to serve as important tools for evaluation and optimization of intratumoral drug delivery. ${ }^{7,8}$ Compared with 2-D studies, spheroids better model in vivo processes by mimicking many of the physiologic characteristics of the native tumor environment, including complex multicellular architecture, barriers to mass transport, and extracellular matrix deposition. ${ }^{9-12}$ Importantly, multicellular spheroid models are more chemoresistant compared with monolayer cells, thus serving as excellent models for the evaluation of drug delivery systems. ${ }^{13}$ Our study builds on previous 2-D monolayer in vitro and in vivo animal studies performed by our group investigating the antitumor efficacy of Pax-eNP. We have utilized a 3-D multicellular mesothelioma spheroid model to investigate the mechanisms of early tumor penetration and prolonged intratumoral drug retention as a means to elucidate the enhanced in vivo efficacy of Pax-eNPs.

\section{MATERIALS AND METHODS \\ NP Preparation}

Pax-eNPs were prepared using a previously described technique loading with 5\% wt/wt paclitaxel (Sigma Aldrich, St. Louis, Mo). ${ }^{1,14}$ For localization studies, eNPs were labeled with rhodamine B (Rho-eNPs) (Polysciences, Inc, Warrington, Pa). For paclitaxel localization studies, eNPs were loaded with standard paclitaxel doped with Oregon Green 488 conjugated paclitaxel (OG-Pax) (Life Technologies Corp, Carlsbad, Calif) at a ratio of 4:1 (ie, Pax:OG-Pax). Free Pax or OG-Pax not contained in eNP was dissolved in Cremophor EL and ethanol (SigmaAldrich) and referred to as Pax-C/E or OG-Pax-C/E, respectively.

\section{Cell Culture and Formation of Spheroids}

Human malignant mesothelioma cells (MSTO-211H; ATCC, Manassas, Va) were grown at $37^{\circ} \mathrm{C}, 5 \%$ carbon dioxide in Roswell Park Memorial Institute 1640 media with $10 \%$ fetal bovine serum, $100 \mu \mathrm{g} / \mathrm{mL}$ streptomycin, and 100 units $/ \mathrm{mL}$ penicillin. To produce MSTO-211H spheroids, 96-well U-bottomed Greiner plates (Sigma-Aldrich, St Louis, Mo) were coated with a $60 \mu \mathrm{L} /$ well of $5 \mathrm{mg} / \mathrm{mL}$ poly-HEMA (poly-2-hydroxyethyl methacrylate; Sigma-Aldrich) in 95\% ethanol and air dried. Monolayer MSTO-211H cells were treated with Accutase (BD Biosciences, San Jose, Calif) for 5 minutes, centrifuged, and replated in poly-HEMAcoated U-bottomed plates at $5 \times 10^{3}$ cells/well in $200 \mu \mathrm{L}$ media. The cells were centrifuged at $216 \mathrm{~g}$ for 10 minutes and incubated at $37^{\circ} \mathrm{C}$ in $5 \%$ carbon dioxide for 24 hours to form spheroids. ${ }^{15,16}$

\section{Assessment of Necrosis and Apoptosis via Fluorescence and Confocal Microscopy}

Spheroid morphology was examined with a Zeiss AxioImagere M1 Microscope (Carl Zeiss Microscopy, Oberkochen, Germany) at 10× magnification with spheroid size determined as the mean of 2 orthogonal diameters.

At each time point, spheroids were washed 3 times with phosphate buffered saline (PBS). Cell nuclei were stained by incubating with 0.2 $\mu \mathrm{g} / \mathrm{mL}$ Hoechst 33342 (blue fluorescent dye; Life Technologies, 


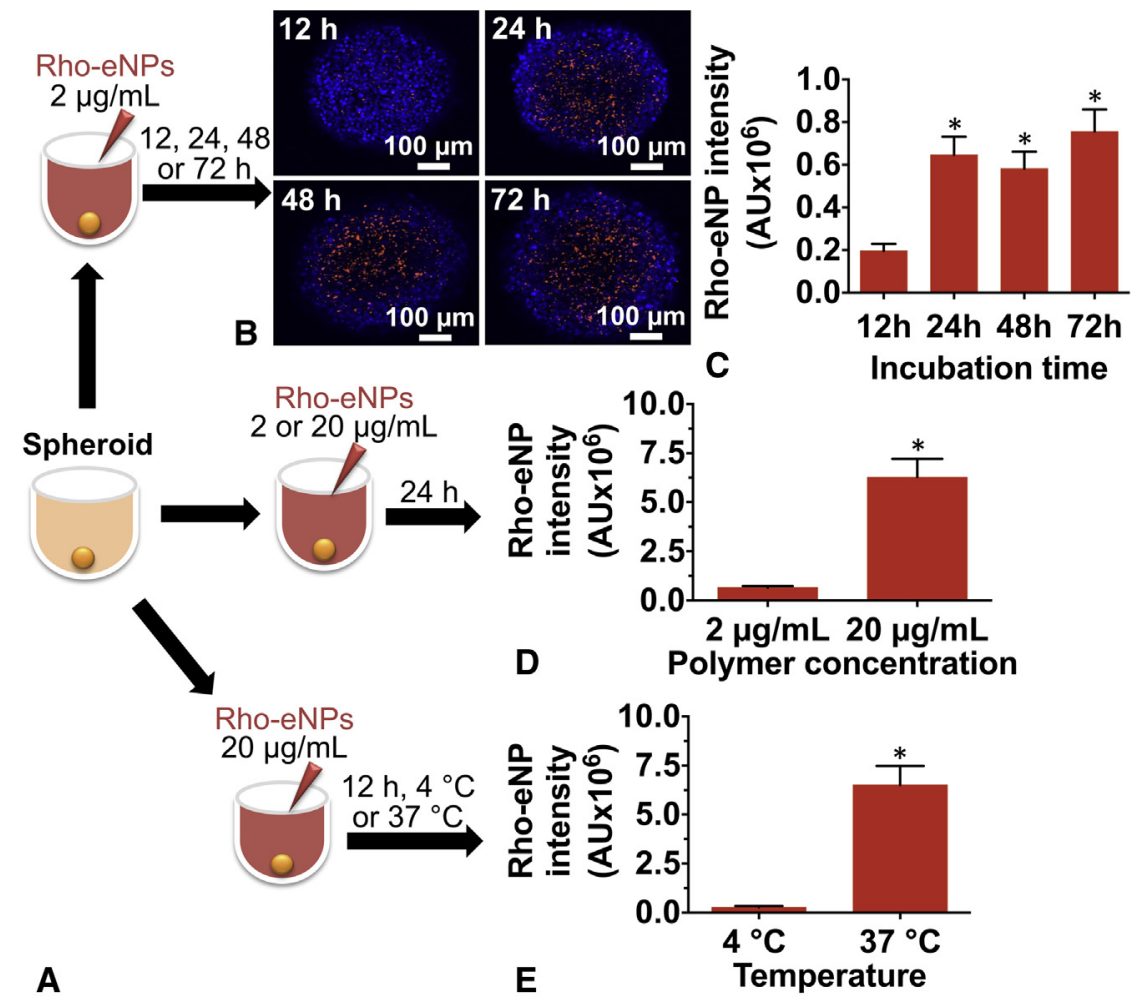

FIGURE 1. Expansile nanoparticles labeled with rhodamine B (Rho-eNP) uptake in mesothelioma tumor spheroids as a function of incubation time, nanoparticle concentration, and temperature as assessed via confocal microscopy. A, Experimental design. B, Representative confocal images of tumor spheroids exposed to Rho-eNPs for 12, 24, 48, and 72 hours. Spheroids with nuclei stained blue with Hoechst 33342 demonstrate increased Rho-eNP uptake (red) over time. C, Rho-eNP intensity (ie, uptake) as a function of time (mean \pm standard error) $(* P=.0014, * P=.0022$, and $* P<.0001$ vs 12 hours, respectively, determined by analysis of variance). D, Rho-eNP uptake in spheroids treated with $2 \mu \mathrm{g} / \mathrm{mL}$ versus $20 \mu \mathrm{g} / \mathrm{mL}$ Rho-eNPs for 24 hours $(* P<.0001 \mathrm{vs} 2 \mu \mathrm{g} / \mathrm{mL}$, determined by $t$ test). E, Rho-eNP uptake in spheroids coincubated at $4^{\circ} \mathrm{C}$ or $37^{\circ} \mathrm{C}\left({ }^{*} P<.0001\right.$ vs $4^{\circ} \mathrm{C}$, determined by $t$ test). $A U$, Arbitrary units.

Carlsbad, Calif) at $37^{\circ} \mathrm{C}$ for 12 minutes. Caspase- 3 activity was detected by adding $2.5 \%(\mathrm{v} / \mathrm{v})$ of Nucview 488 Caspase-3 (Nucview 488 Caspase-3 Assay Kit; Biotium, Hayward, Calif) in media for 45 minutes at $4^{\circ} \mathrm{C}$. Necrosis was assessed by staining with propidium iodide (PI) (Abcam, Cambridge, United Kingdom) $(5 \mu \mathrm{g} / \mathrm{mL})$ for $20 \mathrm{mi}-$ nutes at room temperature. After staining, spheroids were washed 3 times with PBS and transferred to glass-bottom microwell dishes (thickness No. 1.5; MatTek, Ashland, Mass). Spheroids were assessed for necrosis by imaging with a Zeiss LSM 510 inverted confocal laserscanning microscope with Plan-Apochromat $10 \times / 0.45$ lens (Carl Zeiss Microscopy).

When comparing the fluorescence intensity between images, all detection settings of the Zeiss LSM 510 confocal microscope were held constant. All images were taken $75 \pm 5 \mu \mathrm{m}$ from the bottom of spheroids. Spheroids with irregular shape were excluded from analysis. Experiments were repeated at least 3 times with 4 to 8 spheroids at each condition. Fluorescence intensities were quantified using ImageJ with LSM reader plug-ins (version 1.46; National Institutes of Health, Washington, DC).

\section{Rho-eNP Uptake in Spheroids}

Spheroids were treated with Rho-eNPs at concentrations of $2 \mu \mathrm{g} / \mathrm{mL}$ or $20 \mu \mathrm{g} / \mathrm{mL}$, at $4^{\circ} \mathrm{C}$ and $37^{\circ} \mathrm{C}$ and Rho-eNP uptake quantified at $12,24,48$, and 72 hours of incubation. These time points were selected based on previous work published by our group. ${ }^{6,17}$ Tumor spheroids were then washed 3 times with PBS and subsequently analyzed using confocal microscopy.

Spheroids were coincubated with pharmacologic inhibitors of endocytosis, including sodium azide $0.01 \%$, 2-deoxyglucose $20 \mathrm{mM}$, chlorpromazine $10 \mu \mathrm{M}$, hexamethylene amiloride $20 \mu \mathrm{M}$, wortmannin $10 \mu \mathrm{M}$, genistein $50 \mu \mathrm{M}$, and methl- $\beta$-cyclodextrin $5 \mathrm{mM}$. Inhibitor concentrations were based on previously published work. ${ }^{18}$ Following a 1hour preincubation with each endocytic inhibitor, Rho-eNPs $(20 \mu \mathrm{g} / \mathrm{mL})$ were added to the cultures for an additional 4 hours. Spheroids treated with Rho-eNPs without the presence of inhibitors served as positive controls. After 4 hours of Rho-eNP exposure, spheroids were washed 3 times with PBS and imaged using confocal microscopy. Zero rhodamine signal within the spheroid (ie, $100 \%$ inhibition) was used as the positive control. Spheroids cultured with eNP without any inhibitor present were considered negative controls (signal strength defined as $100 \%$ Rho-eNP uptake; that is, $0 \%$ inhibition). All inhibitors were compared with the negative control spheroids ( $0 \%$ inhibition).

\section{Intraspheroidal Delivery of OG-Pax-eNPs}

Spheroids were incubated with $100 \mathrm{ng} / \mathrm{mL}$ OG-Pax-eNPs or OG-Pax-C/ E for 24 hours. Spheroids were then washed with PBS and transferred into paclitaxel-free media for 24 or 72 hours. Confocal microscopy was used to compare intraspheroidal delivery of OG-Pax via eNPs or C/E.

\section{Pax-Rho-eNP-Induced Apoptosis in Tumor Spheroids}

Tumor spheroids were exposed to Pax-Rho-eNPs at $100 \mathrm{ng} / \mathrm{mL}$ paclitaxel concentration for $12,24,48$, or 72 hours. Caspase-3 activity was used to indicate apoptosis. Pax-Rho-eNP intensity was assessed with confocal microscopy to follow eNP accumulation within spheroids. 


\section{Cytotoxicity of Pax-eNPs and Pax-C/E in a Mesothelioma Tumor Spheroid Model}

Spheroids were exposed to Pax-eNPs or Pax-C/E $(100 \mathrm{ng} / \mathrm{mL}$ paclitaxel concentration) for 24 hours; washed; and incubated with fresh media for 24 hours, 72 hours, or 7 days. ${ }^{6}$ In a second, high-dose short-duration experiment, spheroids were treated for 4 hours with Pax-eNPs or Pax-C/E (paclitaxel concentration of $1000 \mathrm{ng} / \mathrm{mL}$ ) and then washed and incubated with fresh media without paclitaxel for 24,48 , or 72 hours. Spheroids were stained with PI as described above.

\section{Statistical Analysis \\ Statistical analysis was performed with GraphPad Prism 6.0 software (La Jolla, Calif). Student $t$ test was used for 2-group comparisons and 1-way analysis of variance with Bonferroni correction (adjusted $P$ values were used) for multiple comparisons to control type I error. For data not in compliance with Gaussian distribution (D'Agostino and Pearson omnibus normality test), nonparametric $t$ test (Kruskal-Wallis test) or anal- ysis of variance (Mann-Whitney $U$ test following Dunn's multiple compar- isons tests) were used. All data are presented as mean \pm standard error of the mean. Exact $P$ values are quoted in the text with $P<.05$ considered to be statistically significant.}

\section{RESULTS \\ Rho-eNP Uptake Within Spheroids: Dose- and Energy-Dependent Macropinocytosis}

Rho-eNP accumulation within tumor spheroids was characterized as a function of time, Rho-eNP concentration, and temperature (Figure 1, A). Rho-eNP accumulation increased with time up to incubation durations of 24 hours (Figure 1, $B$ and $C$ ). Interestingly, there was no significant increase in intraspheroidal accumulation of Rho-eNPs at longer incubation times of 24,48 , or 72 hours. Rho-eNP accumulation was dose-dependent, with 8.5 times greater uptake in spheroids treated with $20 \mu \mathrm{g} / \mathrm{mL}$ versus $2 \mu \mathrm{g} /$ $\mathrm{mL}$ Rho-eNPs $\left(6.28 \times 10^{6}\right.$ AU [arbitrary unit] vs $0.66 \times 10^{6} \mathrm{AU} ; P<.0001$ ) (Figure $1, D$ ). Rho-eNP uptake is an energy dependent process as demonstrated by the significant decrease in Rho-eNP intensity when spheroids were incubated with $20 \mu \mathrm{g} / \mathrm{mL}$ Rho-eNPs for 12 hours at $4^{\circ} \mathrm{C}$ versus $37^{\circ} \mathrm{C}(P<.0001)$ (Figure $\left.1, E\right)$. This dose- and energy-dependent process of eNP uptake was further characterized using endocytotic pharmacologic inhibitors. A 1-hour pretreatment with wortmannin and sodium azide + 2-deoxyglucose resulted in $>50 \%$ inhibition of eNP accumulation within spheroids $(89.8 \% \pm 2.5 \%$ and $77.4 \% \pm$ $13.3 \%$ decrease, respectively; $P<.0001$ ) (Figure 2), confirming that eNP uptake is an energy-dependent process and is consistent with the macropinocytosis pathway.

\section{Greater Intraspheroidal Delivery and Retention of Paclitaxel via eNPs Versus $\mathbf{C} / \mathbf{E}$}

Representative confocal images of tumor spheroids exposed to OG-Pax-eNPs or OG-Pax-C/E revealed significantly greater OG-Pax accumulation after 24 hours of exposure to eNP treatments (Figure 3, A). Quantification of OG-Pax intensity demonstrated 38-fold greater

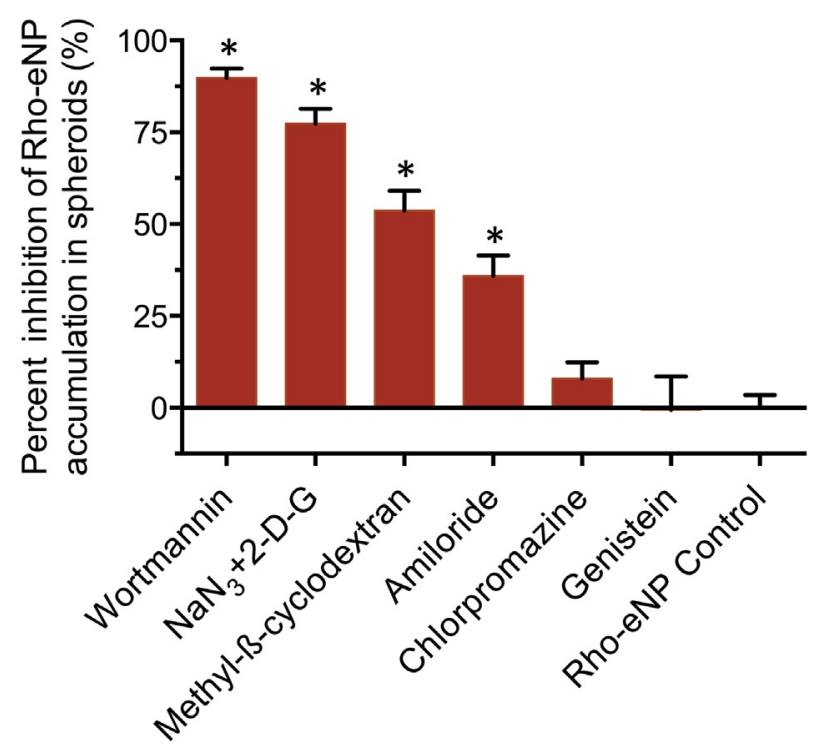

FIGURE 2. Inhibition of expansile nanoparticles labeled with rhodamine $\mathrm{B}($ Rho-eNP) uptake in the presence of endocytotic inhibitors, including the 4 major pathways (clathrin-dependent, caveolaemediated, macropinocytosis, and clathrin- and caveolae-independent endocytosis). Percent Rho-eNP inhibition was measured via confocal microscopy and normalized to noninhibitor controls (mean \pm standard error). $\mathrm{NaN}_{3}+2-\mathrm{D}$ - $\mathrm{G}$, Sodium azide +2 -deoxyglucose. $* P<.0001$ versus Rho-eNP control.

intraspheroidal OG-Pax delivery via eNPs than with an equivalent dose of OG-Pax-C/E $\left(8.85 \times 10^{5} \mathrm{AU}\right.$ vs $0.23 \times 10^{5} \mathrm{AU} ; P<.0001$, identical imaging conditions) (Figure $3, B$ ), indicating that eNPs significantly increase the amount of drug delivered to the spheroid.

Retention of OG-Pax within spheroids was determined by incubating spheroids with OG-Pax-eNPs for 24 hours and then transferring spheroids to fresh media for an additional 24 or 72 hours (ie, a washout period). Intraspheroidal OG-Pax intensity slowly declined after a washout period of 24 or 72 hours (no washout $8.85 \times 10^{5} \mathrm{AU}$ vs $2.20 \times 10^{5} \mathrm{AU}$ and $1.15 \times 10^{5} \mathrm{AU}$, respectively) (Figure 3,C). However even after the 72-hour washout, intraspheroidal OG-Pax intensity in OG-Pax-eNP-treated spheroids was still more than 3 times the peak OG-Pax intensity achieved after OG-Pax-C/E treatment $\left(0.23 \times 10^{5}\right.$ AU) with no washout period. These findings demonstrate that intraspheroidal drug delivery and retention are significantly increased when eNPs are used as the delivery vehicle.

\section{Apoptosis Within Tumor Spheroids Induced by Pax-eNPs}

Spheroids treated with Pax-Rho-eNPs demonstrated caspase-3 upregulation, signaling the induction of apoptosis (Figure 4, A). Caspase-3 activity was negligible within the first 24 hours after Pax-Rho-eNP treatment but significantly 

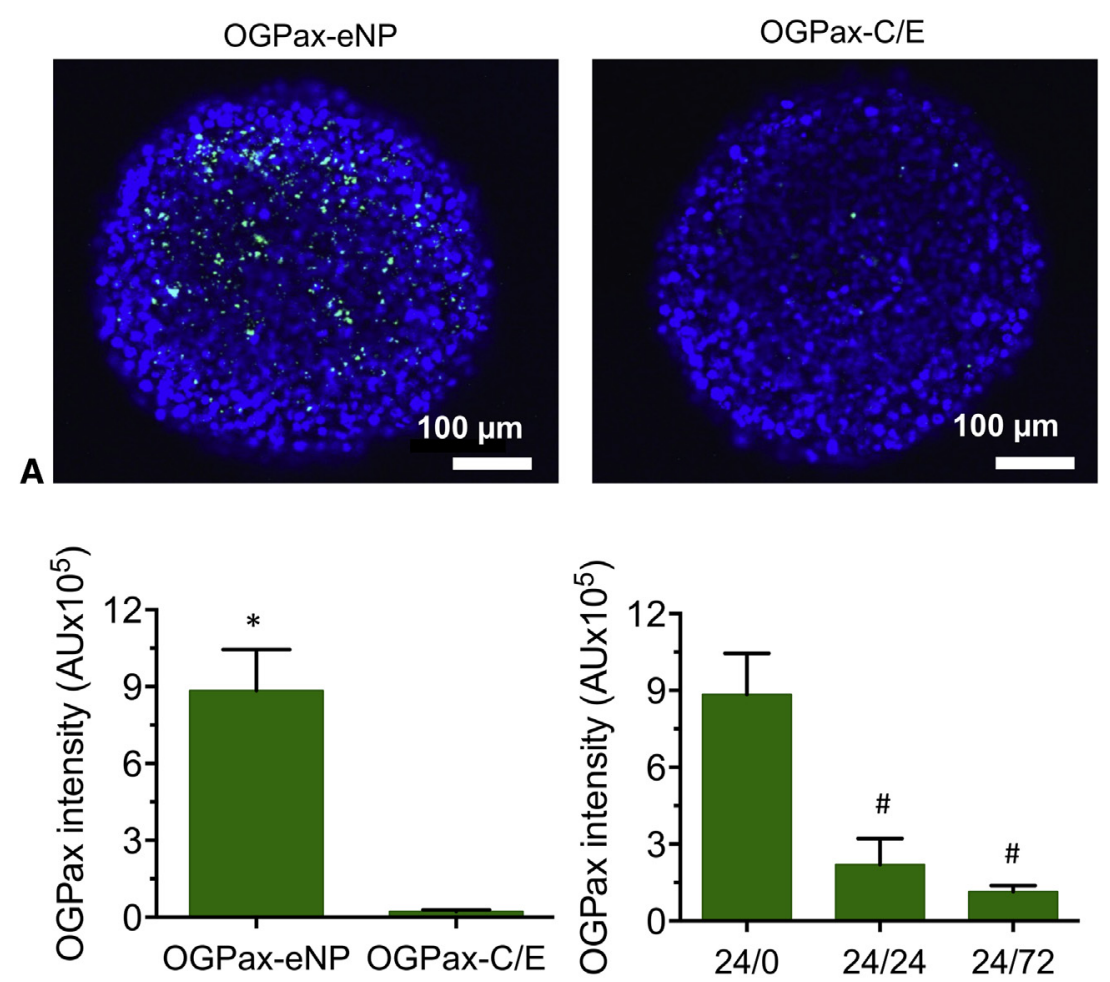

\section{B}

Treatment

C Drug exposure/Washout time (h)

FIGURE 3. Paclitaxel uptake and the kinetics of retention within Oregon Green-conjugated paclitaxel loaded into expansile nanoparticles (OGPax-eNP)treated spheroids. Spheroids were treated with Oregon Green-conjugated paclitaxel dissolved in Cremophor EL/ethanol (OGPax-C/E) (20 ng/mL OGPax) or with the equivalent dose of OGPax-eNPs for 24 hours. A, Confocal images of OGPax-eNPs or OGPax-C/E-treated spheroids with OGPax shown in green. B, OGPax intensity in treated spheroids. C, After 24-hour incubation with OGPax-eNPs, treated spheroids were placed in fresh media and OGPax intensity was measured via confocal microscopy at 0,24 , and 72 hours after washout. All data are mean \pm standard error, Kruskal-Wallis $t$ test, or Mann-Whitney analysis of variance. $24 / 0,24$-hour treatment duration with no posttreatment washout. ${ }^{*} P<.0001$ versus OGPax-C/E. ${ }^{\#} P=.0123$ or $P<.0001$ versus $24 / 0$, respectively. $A U$, Arbitrary units.

increased by 48 hours and 72 hours (17- and 30-fold higher than 24 hours, respectively) (Figure 4, B). This confirmed that paclitaxel delivered via eNPs is released within the spheroids and induces apoptosis throughout the entire cross-section of the spheroid (Figure 4, A).

\section{Pax Delivery via eNPs Yields Increased and Prolonged Cytotoxicity Against Mesothelioma Tumor Spheroids}

Comparison of antitumor efficacy of Pax-eNPs versus Pax-C/E following a 24-hour exposure demonstrated similar PI signal intensity, a marker for necrosis, after 24 hours of treatment and for the first 72 hours after drug washout (Figure 5, A). However, PI intensity continued to increase in the Pax-eNP-treated spheroids over 7 days, whereas PI intensity decreased in Pax-C/E-treated spheroids $(P=.0009)$ (Figure 5, $B)$.

Furthermore, the prolonged effect of Pax-eNPs was present even after a short treatment. Following 4-hour exposure of tumor spheroids to $1000 \mathrm{ng} / \mathrm{mL}$ of either Pax-eNPs or Pax-C/E demonstrated low initial cytotoxicity
(PI intensity) for both treatments through 48-hours of washout. However, a delayed treatment effect was evident in Pax-eNP-treated spheroids (Figure E1), with PI fluorescence increasing from $4.8 \times 10^{5} \mathrm{AU}$ to $8.2 \times 10^{5} \mathrm{AU}$ from the 48- to the 72-hour washout period (Figure 5, B), more than twice the PI intensity exhibited following the equivalent Pax-C/E therapy $\left(8.2 \times 10^{5} \mathrm{AU}\right.$ vs $3.9 \times 10^{5} \mathrm{AU}$; $P=.0030$ ).

\section{DISCUSSION}

The chemoresistance of malignant mesothelioma remains a major therapeutic challenge, with rates of tumor regression reported at $10 \%$ to $30 \%$ for single or combined chemotherapy. ${ }^{19-22}$ The recent observation that local PaxeNP treatment of human intraperitoneal mesothelioma significantly improved survival in an in vivo murine model, compared with free paclitaxel, was striking given that PaxeNP exposure of the identical mesothelioma tumor cells plated as a 2-D monolayer in vitro demonstrated equivalent or inferior antitumor efficacy. ${ }^{6}$ This suggested that eNP may be more effective at penetration and/or persistence within 

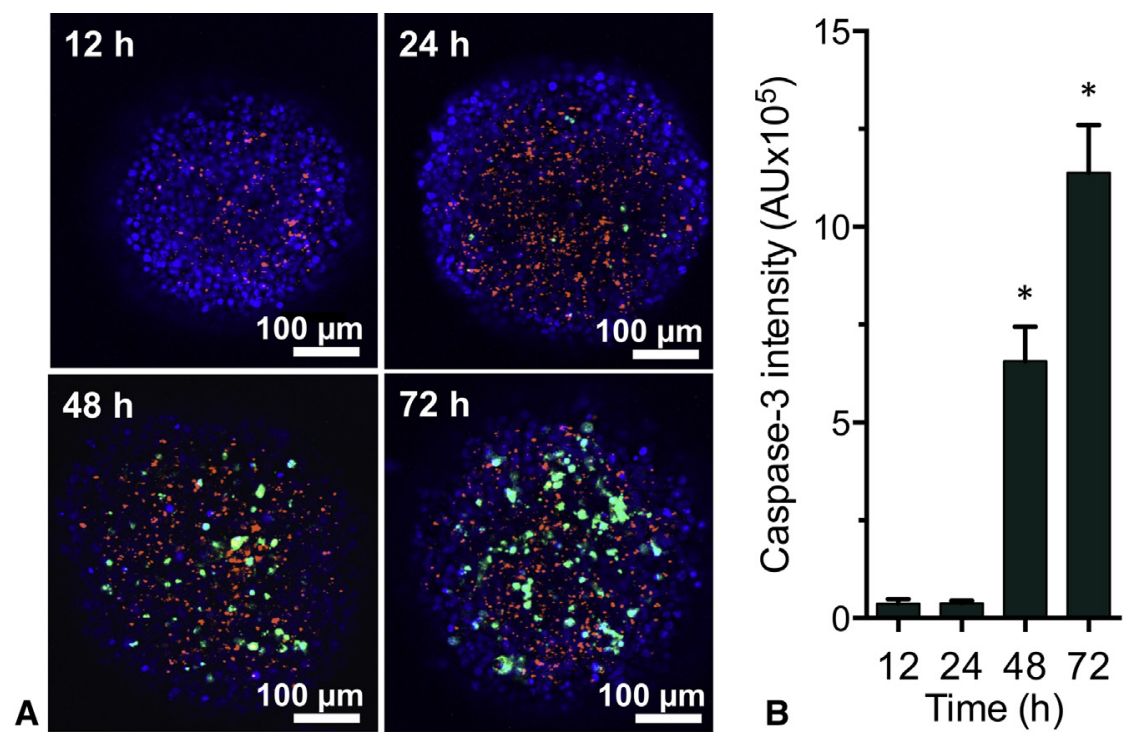

FIGURE 4. The effect of expansile nanoparticles labeled with rhodamine B and paclitaxel (Rho-Pax-eNPs) on caspase- 3 activity in spheroids. A, Spheroids were treated with Rho-Pax-eNPs (paclitaxel concentration $100 \mathrm{ng} / \mathrm{mL}$ ) for 12, 24, 48, and 72 hours. Red represents Rho-eNP localization. Green areas represent activated Caspase-3 and thus apoptotic cells. B, Caspase-3 intensity within spheroids is significantly increased after treatment with Rho-Pax-eNPs over time. $* P<.01$ versus 12 hours and 24 hours, based on Kruskal-Wallis test followed by Dunn's multiple comparisons. $A U$, Arbitrary units.

multicellular tumors and led to the use of a 3-D tumor spheroid model to further elucidate the mechanisms of Pax-eNP-enhanced tumor cytotoxicity and explore the superior efficacy noted in more complex tumor structures in vivo. The results demonstrate that enhanced tumor penetration and prolonged intratumoral drug release are unique mechanisms of Pax-eNP cytotoxicity. Given the parallels with native tumor physiology, these findings suggest that 3-D spheroids may be a valuable tool to elucidate the cytotoxic mechanisms and NP-tumor interactions that NPs leverage for greater antitumor efficacy in vivo.

To achieve an effective chemotherapeutic response, NPs must penetrate and accumulate drug within the tumor. ${ }^{23-27}$ Using a 3-D malignant mesothelioma spheroid model we have better characterized this process, demonstrating that tumor penetration by Pax-eNPs is time-, dose-, and temperature(energy) dependent and is significantly enhanced within multicellular tumor structures. Whereas others have investigated NP penetration in various spheroid tumor models, ${ }^{25,26} \mathrm{NP}$ retention after a washout period, akin to clearance in vivo, and the effect on intraspheroidal drug delivery, have not previously been assessed. Consistent with prior results for Pax-eNP-treated tumors in vivo, Pax-eNPs deliver significantly more paclitaxel within tumor spheroids than Pax-C/E alone, and are retained within spheroids for a significantly longer duration, thereby increasing intratumoral delivery of paclitaxel and facilitating prolonged antitumor cytotoxicity. Furthermore, rapid intraspheroidal delivery and prolonged retention of Pax-eNPs leads to increased apoptosis even a week after drug washout. These findings suggest that the superior efficacy of Pax-eNPs against chemoresistant malignant mesothelioma in vivo is secondary to enhanced tumor penetration and retention within multicellular tumor structures, coupled with the subsequent slow release of paclitaxel over time.

Using pharmacologic endocytosis inhibitors to block various cellular uptake pathways, Zubris and colleagues ${ }^{18}$ identified macropinocytosis as the major cellular pathway responsible for eNP uptake by breast cancer cells within monolayer cultures. Macropinocytosis is a specific form of endocytosis that is initiated by the transient activation of receptor tyrosine kinases and results in the formation of large intracellular vacuoles called macropinosomes. ${ }^{28,29}$ Using these same specific pharmacologic inhibitors, we examined the dominant endocytotic pathway responsible for intracellular uptake of eNPs within spheroids. Wortmannin, a phosphoinositide 3-kinase inhibitor; hexamethylene amiloride, a selective ion channel inhibitor; and methyl- $\beta$-cyclodextrin, an inhibitor of cholesteroldependent endocytosis, were all shown to significantly inhibit Rho-eNP uptake by spheroids compared with untreated controls. Given that phosphoinositide 3-kinase inhibition is integral to cytoskeletal remodeling and macropinocytosis, ${ }^{30}$ and hexamethylene amiloride and methyl- $\beta$-cyclodextrin are both involved in the same endocytotic pathway, the dominant endocytotic pathway for eNP intracellular uptake within spheroids also appears to be macropinocytosis, suggesting a central mechanism for eNP tumor penetration in vivo. Furthermore, the inhibition of Rho-eNP uptake by sodium azide +2 -deoxyglucose and at a lower temperature $\left(4^{\circ} \mathrm{C}\right)$, confirms that eNP uptake does not occur passively but is an energy-dependent process, as has been previously reported. ${ }^{25}$ Although the mechanism of eNP uptake is similar for 

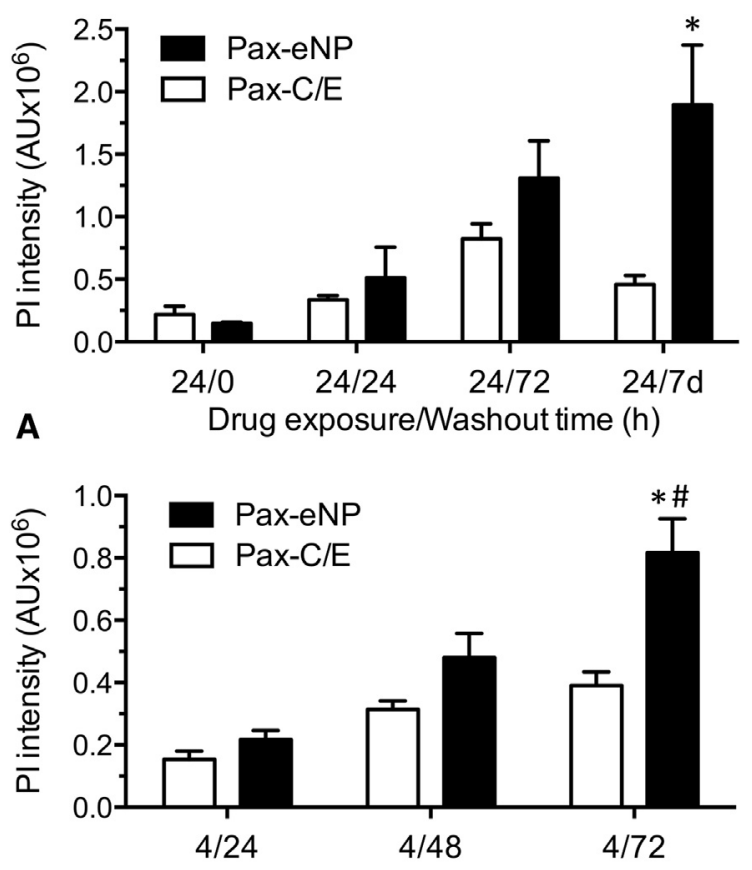

B

Drug exposure/Washout time $(\mathrm{h})$

FIGURE 5. The effect of paclitaxel expansile nanoparticles (Pax-eNPs) and paclitaxel Cremophor EL/ethanol $(\mathrm{Pax}-\mathrm{C} / E)$ on intraspheroidal tumor cytotoxicity. A, Propidium iodide $(P I)$ signal intensity within spheroids after 24-hour exposure to Pax-eNPs or Pax-C/E at $100 \mathrm{ng} / \mathrm{mL}$. Spheroids treated for 24 hours and imaged immediately are designated 24/0, whereas $24 / 24,24 / 72$, and 24/7d represent spheroids treated for 24 hours with measurement of PI intensity at 24 hours, 72 hours, or 7 days after washout, respectively. $* P=.0009$ versus Pax-C/E 24/7d. Note: Paclitaxel concentration was $100 \mathrm{ng} / \mathrm{mL}$ (lower dose). B, PI signal intensity after spheroid exposure to Pax-eNPs or Pax-C/E (1000 ng/mL) for 4 hours, followed by drug-free media culture for 24,48 , and 72 hours. $* P=.0030$ versus Pax-C/E 4/72 based on Mann-Whitney $U$ test. ${ }^{\#} P=.0002$ versus Pax-eNP 4/24 based on Kruskal-Wallis test. $A U$, Arbitrary units.

individual tumor cells within a 2-D monolayer or a 3-D spheroid, the persistence of OG-Pax deep within spheroids and the resulting prolonged drug release are unique to PaxeNP delivery.

Lastly, the prolonged tumor cytotoxicity resulting from short-term Pax-eNP exposure has major clinical relevance. Clinically, paclitaxel is administered over a 3- to 4-hour period with intratumoral paclitaxel accumulation reaching a maximum at 3 hours. ${ }^{19}$ However, the cell cycle specificity of paclitaxel delays the onset of tumor apoptosis and rapid clearance of paclitaxel from the circulation significantly limits tumor exposure to therapeutic drug levels, thereby lowering clinical efficacy. ${ }^{31-33}$ Therefore, to mimic current clinical delivery, we investigated spheroid cytotoxicity following short-term (ie, 4 hour) high-dose (ie, $1000 \mathrm{ng} /$ $\mathrm{mL}$ ) paclitaxel exposure. Interestingly, compared with Pax-C/E, a 4-hour Pax-eNP exposure resulted in significantly greater tumor cytotoxicity at 72 hours. Continued cytotoxicity 7 days later is due to the fact that Pax-eNPs rapidly enter the tumor spheroid and remain intracellular, slowly releasing the drug as eNPs expand in response to endosomal $\mathrm{pH}$. The prolonged drug-release mechanism used by $\mathrm{pH}$-triggered Pax-eNP appears to be unique, leading to markedly higher intraspheroidal drug delivery, prolonged intratumoral drug release, and superior antitumor efficacy.

Although 3-D spheroids do model critical physiologic parameters present in vivo, including complex multicellular architecture, barriers to mass transport, and extracellular matrix deposition, ${ }^{12}$ we acknowledge that this study has a number of limitations. Most importantly, the current 3-D cell culture model makes quantitative assessment difficult without disrupting the spheroid structure and multiple cell types commonly present within tumors are not included, thereby limiting our ability to effectively replicate the tumor microenvironment. Future studies are underway to investigate the role of tumor macrophages in eNP uptake within co-cultured spheroid models and further elucidate how multiple cell types within a tumor can influence drug delivery and efficacy in vivo.

\section{CONCLUSIONS}

Compared with standard monolayer cell culture, the in vitro 3-D mesothelioma spheroid model better reflects the increased tumor cytotoxicity demonstrated by PaxeNPs in vivo. The results support our hypothesis that the superior efficacy of Pax-eNPs demonstrated in vivo is secondary to early, persistent tumor penetration and prolonged intratumoral drug release. 3-D multicellular tumor spheroid models are useful tools to investigate cytotoxic mechanisms and nanoparticle-tumor interactions to more accurately assess in vivo cytotoxic activity and clinical outcomes. Given the high cost and limitations of in vivo animal studies, spheroid models may present a clinically relevant platform for screening novel pharmaceuticals and unique drug-delivery systems during the preclinical phase.

\section{Conflict of Interest Statement}

Mark W. Grinstaff reports consulting fees from and equity ownership in AcuityBio Inc, as well as equity ownership in Hyperbranch Medical Tech. All other authors have nothing to disclose with regard to commercial support.

You can watch a Webcast of this AATS meeting presentation by going to: http://webcast.aats.org/2014/files/ Tuesday/20140429_720AM_730AM_Hongyi_Lei.mp4.

The authors thank the Confocal Core Facility staff at Beth Israel Deaconess Medical Center under the direction of Lay-Hong Ang for their contributions.

\section{References}

1. Griset AP, Walpole J, Liu R, Gaffey A, Colson YL, Grinstaff MW. Expansile nanoparticles: synthesis, characterization, and in vivo efficacy of an acidresponsive polymeric drug delivery system. J Am Chem Soc. 2009;131:2469-71. 
2. Peer D, Karp JM, Hong S, Farokhzad OC, Margalit R, Langer R. Nanocarriers as an emerging platform for cancer therapy. Nature Nanotechnol. 2007;2:751-60.

3. Sahoo SK, Labhasetwar V. Nanotech approaches to drug delivery and imaging. Drug Discov Today. 2003;8:1112-20.

4. Vijayaraghavalu S, Raghavan D, Labhasetwar V. Nanoparticles for delivery of chemotherapeutic agents to tumors. Curr Opin Investig Drugs. 2007;8:477-84.

5. Zhang G, Zeng X, Li P. Nanomaterials in cancer-therapy drug delivery system. $J$ Biomed Nanotechnol. 2013:9:741-50.

6. Colson YL, Liu R, Southard EB, Schulz MD, Wade JE, Griset AP, et al. The performance of expansile nanoparticles in a murine model of peritoneal carcinomatosis. Biomaterials. 2011;32:832-40.

7. Kim B, Han G, Toley BJ, Kim CK, Rotello VM, Forbes NS. Tuning payload delivery in tumour cylindroids using gold nanoparticles. Nature Nanotechnol. 2010;5:465-72.

8. Minchinton AI, Tannock IF. Drug penetration in solid tumours. Nat Rev Cancer. 2006;6:583-92.

9. Birgersdotter A, Sandberg R, Ernberg I. Gene expression perturbation in vitro-a growing case for three-dimensional (3D) culture systems. Semin Cancer Biol. 2005; $15: 405-12$

10. Dufau I, Frongia C, Sicard F, Dedieu L, Cordelier P, Ausseil F, et al. Multicellular tumor spheroid model to evaluate spatio-temporal dynamics effect of chemotherapeutics: application to the gemcitabine/CHK1 inhibitor combination in pancreatic cancer. BMC Cancer. 2012;12:15.

11. Fallica B, Makin G, Zaman MH. Bioengineering approaches to study multidrug resistance in tumor cells. Integrat Biol. 2011;3:529-39.

12. Sutherland RM, McCredie JA, Inch WR. Growth of multicell spheroids in tissue culture as a model of nodular carcinomas. J Natl Cancer Inst. 1971;46:113-20.

13. Mehta G, Hsiao AY, Ingram M, Luker GD, Takayama S. Opportunities and challenges for use of tumor spheroids as models to test drug delivery and efficacy. $J$ Control Release. 2012;164:192-204.

14. Colby AH, Colson YL, Grinstaff MW. Microscopy and tunable resistive pulse sensing characterization of the swelling of $\mathrm{pH}$-responsive, polymeric expansile nanoparticles. Nanoscale. 2013;5:3496-504.

15. Friedrich J, Seidel C, Ebner R, Kunz-Schughart LA. Spheroid-based drug screen: considerations and practical approach. Nature Protoc. 2009;4:309-24.

16. Huo S, Ma H, Huang K, Liu J, Wei T, Jin S, et al. Superior penetration and retention behavior of $50 \mathrm{~nm}$ gold nanoparticles in tumors. Cancer Res. 2013;73:319-30.

17. Gilmore D, Schulz M, Liu R, Zubris KA, Padera RF, Catalano PJ, et al. Cytoreductive surgery and intraoperative administration of paclitaxel-loaded expansile nanoparticles delay tumor recurrence in ovarian carcinoma. Ann Surg Oncol. 2013;20:1684-93.

18. Zubris KA, Liu R, Colby A, Schulz MD, Colson YL, Grinstaff MW. In vitro activity of Paclitaxel-loaded polymeric expansile nanoparticles in breast cancer cells. Biomacromolecules. 2013;14:2074-82.

19. Tomek S, Emri S, Krejcy K, Manegold C. Chemotherapy for malignant pleural mesothelioma: past results and recent developments. Br J Cancer. 2003;88:167-74.

20. Van Meerbeeck J, Debruyne C, van Zandwijk N, Postmus PE, Pennucci MC, van Breukelen F, et al. Paclitaxel for malignant pleural mesothelioma: a Phase II study of the EORTC Lung Cancer Cooperative Group. Br J Cancer. 1996;74:961-3.

21. Ellis P, Davies AM, Evans WK, Haynes AE, Lloyd NS, Lung Cancer Disease Site Group of Cancer Care Ontario's Program in Evidence-based Care. The use of chemotherapy in patients with advanced malignant pleural mesothelioma: a systematic review and practice guideline. J Thorac Oncol. 2006;1:591-601.

22. Linden CJ, Mercke C, Albrechtsson U, Johansson L, Ewers SB. Effect of hemithorax irradiation alone or combined with doxorubicin and cyclophosphamide in 47 pleural mesotheliomas: a nonrandomized phase II study. Eur Respir J. 1996;9: 2565-72.

23. Hamilton G. Multicellular spheroids as an in vitro tumor model. Cancer Lett. 1998;131:29-34.

24. Zhen X, Wang X, Xie C, Wu W, Jiang X. Cellular uptake, antitumor response and tumor penetration of cisplatin-loaded milk protein nanoparticles. Biomaterials. 2013:34:1372-82.

25. Gu G, Gao X, Hu Q, Kang T, Liu Z, Jiang M, et al. The influence of the penetrating peptide iRGD on the effect of paclitaxel-loaded MT1-AF7p-conjugated nanoparticles on glioma cells. Biomaterials. 2013;34:5138-48.

26. Wang X, Zhen X, Wang J, Zhang J, Wu W, Jiang X. Doxorubicin delivery to 3D multicellular spheroids and tumors based on boronic acid-rich chitosan nanoparticles. Biomaterials. 2013;34:4667-79.

27. Liu R, Gilmore DM, Zubris KA, Xu X, Catalano PJ, Padera RF, et al. Prevention of nodal metastases in breast cancer following the lymphatic migration of paclitaxel-loaded expansile nanoparticles. Biomaterials. 2013;34: 1810-9.
28. Falcone S, Cocucci E, Podini P, Kirchhausen T, Clementi E, Meldolesi J. Macropinocytosis: regulated coordination of endocytic and exocytic membrane traffic events. J Cell Sci. 2006;119(Pt 22):4758-69.

29. Kerr MC, Teasdale RD. Defining macropinocytosis. Traffic. 2009;10:364-71.

30. Rupper A, Lee K, Knecht D, Cardelli J. Sequential activities of phosphoinositide 3-kinase, PKB/Aakt, and Rab7 during macropinosome formation in dictyostelium. Mol Biol Cell. 2001;12:2813-24.

31. Au JL, Li D, Gan Y, Gao X, Johnson AL, Johnston J, et al. Pharmacodynamics of immediate and delayed effects of paclitaxel: role of slow apoptosis and intracellular drug retention. Cancer Res. 1998;58:2141-8.

32. Desai N, Trieu V, Yao Z, Louie L, Ci S, Yang A, et al. Increased antitumor activity, intratumor paclitaxel concentrations, and endothelial cell transport of cremophor-free, albumin-bound paclitaxel, ABI-007, compared with cremophor-based paclitaxel. Clin Cancer Res. 2006;12:1317-24.

33. Sparreboom A, Scripture CD, Trieu V, Williams PJ, De T, Yang A, et al. Comparative preclinical and clinical pharmacokinetics of a cremophor-free, nanoparticle albumin-bound paclitaxel (ABI-007) and paclitaxel formulated in Cremophor (Taxol). Clin Cancer Res. 2005;11:4136-43.

Key Words: mesothelioma, spheroids, nanoparticle, paclitaxel

\section{Discussion}

Dr Joseph Friedberg (Philadelphia, Pa). Yolonda, I congratulate you on this innovative work. I think there is a lot to it. I think the spheroids are interesting because they have an oxygen gradient that I think is probably much more reflective than a monolayer where you are going to have uniform oxygen. Obviously we know that a lot of the things that we do are oxygen-dependent.

Before I ask my questions, I just want some clarification on the technique. When you say you gave an equivalent drug dose, it's the same concentration of drug, but 1 is delivered free and then 1 is contained within the nanoparticles, and it's just 1 shot? You put them in at the same time?

Dr Colson. Correct.

Dr Friedberg. Have you done the positive control where you separate the free drug, giving it in smaller doses over a longer period of time? Has that ever been done?

Dr Colson. We didn't do it in the spheroid model, but we have done it in vivo in the animal models. The data that I showed you were multiple doses, with the idea that we could get a level up that would stay. What we found was that all of the animals died during the treatment phase because mesothelioma is resistant to paclitaxel, whereas we got through the whole treatment and it was much longer when we gave repetitive doses of the nanoparticles. Even if you give a larger dose, it doesn't catch up.

Dr Friedberg. Have you done it in vitro with the spheroid model? It's clear that you have an active pinocytotic mechanism - the drug is getting in—so you have that, but before I would be convinced that it is superior, at least in the spheroid model, I would want to see several—so if you are giving $100 \mathrm{mg}$ all together, or whatever, and it's 1 shot with the nanoparticles, to give maybe 5 times 20 or whatever.

Dr Colson. Just break it out so you get a constant level?

Dr Friedberg. Yes, break it out. Have you done that?

Dr Colson. We haven't done lower doses constantly yet.

Dr Friedberg. More like we give chemotherapy in patients, I would think, to make the leap that this is more reflective of the in vivo.

Dr Colson. Right.

Dr Friedberg. How big are the nanoparticles? 
Dr Colson. We can make them different sizes. That is why I am hesitating a little bit. We try to get them probably around $50 \mathrm{~nm}$ or so.

Dr Friedberg. When you did it in vivo, was it given intravascularly?

Dr Colson. We gave it via intraperitoneal injection. One can coat nanoparticles a little bit, but when administered intravascularly they get such a big uptake in the lung and the spleen that it's hard to calculate what your dose really is. For this model, we gave the doses all intraperitoneally and they seemed to home right to the tumor.

Dr Friedberg. Clinically, would you picture giving these locally or are you capitalizing on the size within the abnormal vasculature in a tumor for delivery?

Dr Colson. We think, at least for now, that the idea of giving them locally for these diseases would have much higher effi- cacy, and we tend to get them so that they are local, within that area.

Dr Friedberg. Have you done a biodistribution? When you give the dose intraperitoneally, do you find it in other places in the body?

Dr Colson. It tends to stay very localized. We can get it somewhat in the lymphatic system to the lymph nodes, so we have been able to show that we can traffic it that way. In another model it actually decreased lymph node metastases, but we haven't been able to find it in the liver and spleen and some of the other areas where we are more worried about.

Dr Friedberg. It doesn't show up in other organs?

Dr Colson. We don't seem to see that.

Dr Friedberg. That's great.

Readers who found these articles interesting may also like to read the following papers found in recent and future issues of our sister publications, Seminars in Thoracic and Cardiovascular Surgery and Operative Techniques in Thoracic and Cardiovascular Surgery!

News and Views: John Ikonomidis. Integrated Surgical Residency Initiative: Implications For Cardiothoracic Surgery. Semin Thorac Cardiovasc Surg. Spring 2014;26(1):14-26.

Discussion in Cardiothoracic Treatment and Care: Richard Shemin, David Fullerton, John Ikonomidis, John Mayer, Cameron Wright. Manpower. Semin Thorac Cardiovasc Surg. Expected publication December 2014. 

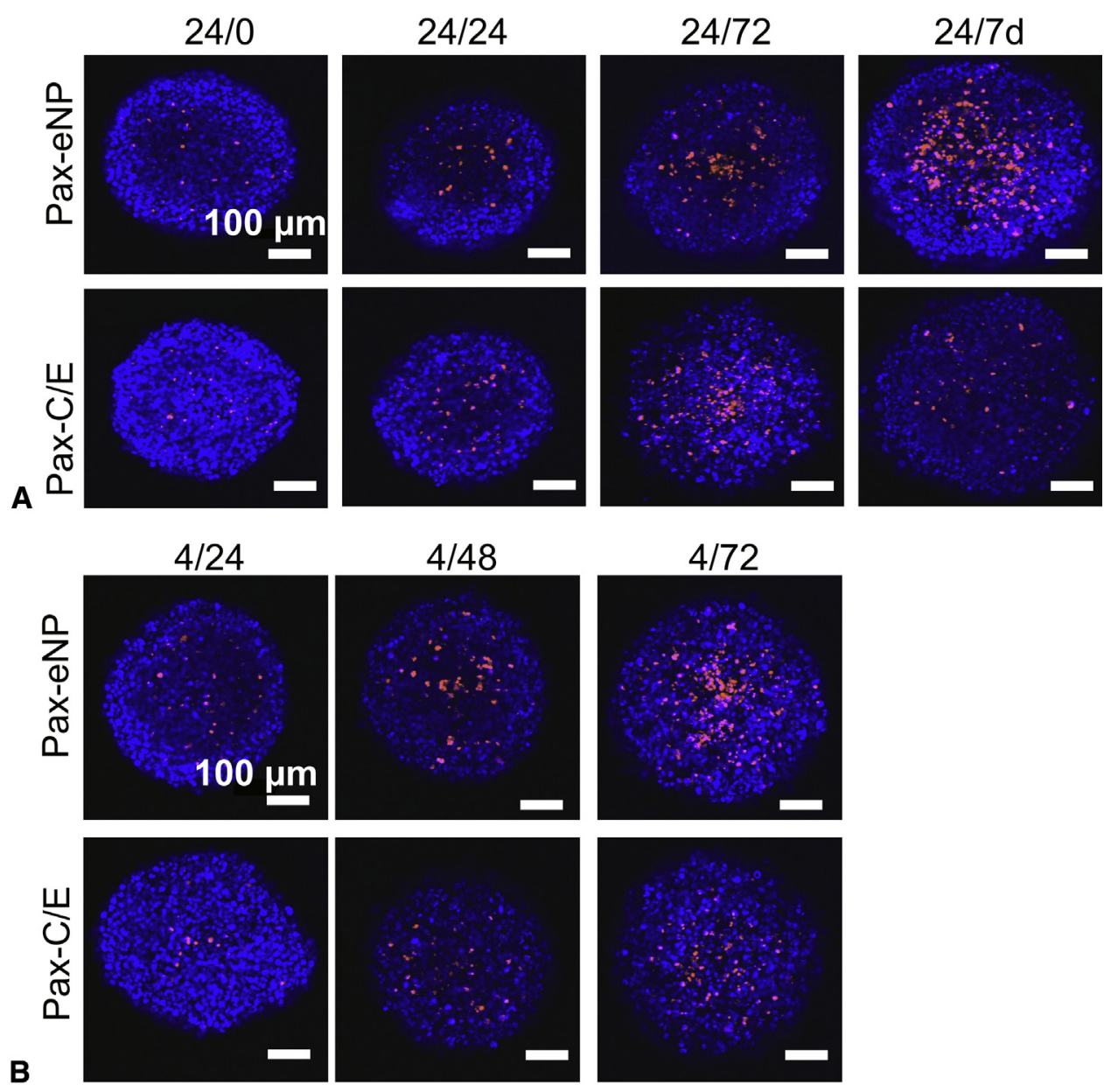

FIGURE E1. A, The effect of Pax-eNP and Pax-C/E on PI intensity in spheroids. Green represents OG-Pax, and red represents PI (necrosis). Spheroids treated with Pax-eNP or Pax-C/E for 24 hours, then washed and exchanged fresh media 24 hours, 48 hours, 72 hours, or 7 days. Spheroids treated for 24 hours and imaged immediately are designated 24/0, while 24/24, 24/72, and 24/7d represent spheroids treated for 24 hours, with measurement of PI intensity 24 hours, 72 hours, or 7 days later, respectively. B,The enhanced delayed effect of paclitaxel-induced spheroids cytotoxicity using Pax-eNP delivery. Red represents PI. Spheroids were exposed with Pax-eNP or Pax-C/E for 4 hours, exchanged fresh media for 24 hours, 48 hours, and 72 hours and underwent confocal imaging for evidence of non-viable cell using PI. PI is shown in red with cell nuclei in blue. 